Background Manuals and Toolkits (MT) are standards for developing Clinical Practice Guidelines (CPG). Most developers have their own MT. There isn't enough information about characteristics of MT in other languages than English.

Objective To assess the characteristics of MT for developing CPG from different developers in English and Spanish.

Methods We searched electronic databases, national clearinghouses and non-electronic sources such as guidelines developer's sites. Epidemiologists independently assessed MT retrieved. Information about scoping, development group, Conflict of Interests (COI), updating, evidence systems among others, were extracted. Results Twenty MT were retrieved, 8 in Spanish, and 12 in English. It is not clear how COI is declared and handled in most of the MT. GRADE and SIGN were the most recommended systems for assessment of quality of evidence, nevertheless many didn't recommend any system. Only 2 MT had a complete explanation about patient's participation. Three years is the most common recommendation for updating CPG. Only a few include an economic component. There isn't clarity in how recommendations are reported and how should be the external review of MT.

Discussion There is heterogeneity in CPG development. Spanish MT are less specific than English ones. It is important to improve quality of Spanish-language MT's, in order to enhance quality of Spanish CPG. There is an important lack of information about patient's participation and drafting of recommendations.

Implications for Guideline Developers/Users It's important to improve the contents and quality of MT in order to achieve high quality standards on CPG development for both developed and developing countries.

\section{P070 TOOLBOX FOR THE COMPLETE PROCESS OF GUIDELINE DEVELOPMENT, REVISION, IMPLEMENTATION AND EVALUATION}

M Hilbink, M Ouwens, T Kool. Radboud University Nijmegen Medical Centre, Nijmegen, The Netherlands

\section{0:1136/bmjqs-2013-002293.148}

Background Problems in the process of guideline development, revision, implementation and evaluation are commonly perceived. Objectives To support and improve the process of guideline development, revision, implementation and evaluation.

Methods After reaching consensus about topics for which there was a huge need for support, we composed thirteen working groups consisting of 4-5 representatives of various Dutch institutions involved in guideline development and implementation. Each group developed a support tool on a specific topic. 150 experts commented the draft version of the tools. Subsequently, the tools were used in more than 40 guideline projects to evaluate their practical value. The final versions of the tools have been disseminated by internet and will be adopted by the National Dutch Quality Insitute.

Results A toolbox containing 13 tools on the following topics: 1. Analysis of clinical care gaps 2. Cost-effectiveness 3. Organization and cooperation 4. Dealing with conflicts 5. International cooperation 6 . Project management 7 . Formulating specific recommendations 8. Attention for sex differences 9. Guidelines and shared decision making 10. Knowledge gaps 11. Implementation 12. Monitoring 13. Electronic disclosure A both Dutch and Englishlanguage version website on guideline development and implementation in the broader context, with incorporation of the tools. Discussion This project yielded a toolbox with tools on topics and activities that offered scope for further international development.
Implications for Guideline Developers/Users Using these tools might improve the quality of guidelines, which in turn results in higher guideline adherence. Better guideline adherence might eventually lead to improved quality of care.

\section{P071 GUIDELINES FOR GUIDELINE DEVELOPERS: A SYSTEMATIC REVIEW OF GRADING SYSTEMS FOR MEDICAL TESTS}

${ }^{1} \mathrm{G}$ Gopalakrishna, ${ }^{2} \mathrm{M}$ Langendam, ${ }^{2} \mathrm{R}$ Scholten, ${ }^{1} \mathrm{P}$ Bossuyt, ${ }^{1} \mathrm{M}$ Leeflang. ${ }^{1}$ Department of Clinical Epidemiology, Biostatistics and Bioinformatics. Academic, Amsterdam, Netherlands; ${ }^{2}$ Dutch Cochrane Centre. Academic Medical Center. Amsterdam Netherlands, Amsterdam, Netherlands

\section{0:1136/bmjqs-2013-002293.149}

Background Development of guidelines for medical tests are challenging given the indirectness of evidence on patient outcomes. We compared grading systems for medical tests in terms of basic guideline quality requirements and on how they use indirect evidence.

Methods We used a systematic search to identify grading systems specific to medical tests in PubMed, professional guideline websites and handsearching back references of key articles. Using the AGREE instrument as a starting point, we defined two sets of characteristics to describe these systems: process and methodological ones. Process characteristics were features related to the guideline development process. Methodological characteristics were defined as features relating to how evidence is gathered, appraised and recommendations development. Data was extracted in duplicate and differences resolved through discussion.

Results Twelve grading systems were included. Process characteristics least often addressed were whether the system was piloted (3/12) and funder information (3/12). Methodologically, developing a clinical scenario, care pathway and/or analytical framework, having explicit criteria for appraising and linking indirect evidence, and having explicit methodologies for translating evidence into recommendations were least frequently addressed. Five systems at most addressed these to varying degrees of completeness.

Implications for Guideline Developers There is a need for standardisation of basic guideline features a grading system should address. No one system adequately addressed the complexity of gathering, assessing and linking different bodies of evidence. There is a need for critical appraisal of these features in each system and for targeted user testing among guideline developers.

\section{P081 DESIGN OF PHYSICIAN PRINTED EDUCATIONAL MATERIALS: MAKING GOOD IDEAS STICK}

J Versloot, M Kastner, A Grudniewicz, A Chatterjee, L Hayden, O Bhattacharyya. St. Michael's Hospital, Toronto, Canada

\section{0:1136/bmjas-2013-002293.150}

Background It is difficult to communicate new and complex clinical evidence to physicians already experiencing information overload. Proper use of design principles may increase uptake of guidelines and other printed educational materials (PEM) and improve practice.

Objectives We aimed to determine whether physician-oriented PEMs are created in accordance with design principles.

Methods We analysed PEMs identified in a 2012 Cochrane review of their effect on professional and patient outcomes and developed a checklist of design principles based on a literature 